\title{
Two-frequency emission and polarization dynamics at lasing threshold in vertical-cavity surface-emitting lasers
}

\author{
M. Sondermann, M. Weinkath, and T. Ackemann \\ Institut für Angewandte Physik, Westfälische Wilhelms-Universität Münster, Corrensstrasse 2/4, D-48149 Münster, Germany \\ J. Mulet and S. Balle \\ Instituto Mediterráneo de Estudios Avanzados (IMEDEA), Universitat de les Illes Balears, E-07071 Palma de Mallorca, Spain
}

(Received 14 October 2002; published 29 September 2003)

\begin{abstract}
We investigate experimentally and theoretically the polarization dynamics in vertical-cavity surface-emitting lasers in the vicinity of the lasing threshold. If the net gain anisotropy between the two orthogonally polarized modes is close to zero, the laser operates in a partially polarized state. This state occurs at and slightly above the lasing threshold. It is characterized by anticorrelated dynamics of the two modes which result from completely anticorrelated dynamics at low frequencies and correlated dynamics on the time scale of the relaxation oscillations. If the net anisotropy is changed by a change of the detuning between the cavity resonance and the gain maximum, one of the two linear polarization states is selected depending on the sign of the net gain. For one sign the well-known phenomenon of polarization switching is recovered at higher currents. The experimental results show very good agreement with simulations based on a model that takes into account nonlinear spin dynamics and linear temperature-dependent detuning effects.
\end{abstract}

DOI: 10.1103/PhysRevA.68.033822

PACS number(s): 42.55.Px, 42.60.Mi, 42.65.Sf

\section{INTRODUCTION}

The polarization dynamics in vertical-cavity surfaceemitting lasers (VCSELs) has been subject of many experimental [1-7] and theoretical [8-14] studies over the last few years. Unlike in edge-emitting semiconductor lasers, VCSELs allow for one longitudinal mode only due to the short cavity length. A further difference, which has a strong impact on the polarization properties of this devices, is that the circular geometry of the VCSEL cavity does not fix the polarization direction of the emitted light. Nevertheless, in most cases one linearly polarized fundamental transverse mode is selected at the lasing threshold [1-6]. This is due to linear polarization anisotropies, i.e., dichroism and birefringence, which are induced by unavoidable mechanical stress via the elasto-optic effect $[1,15]$ and by the electro-optic effect [16]. The birefringence leads to a removal of the frequency degeneracy of modes with different polarization and thus to a frequency splitting. Due to the dichroism these modes experience a different net gain (net gain=unsaturated gain - unsaturated losses), which leads to a selection of one polarization mode at the threshold [1]. For simplicity, we use the term dichroism for the net gain anisotropy resulting from various effects such as mechanical stress $[1,5,15]$, anisotropic losses in the Bragg mirrors of the VCSELs [17], and gain differences for the polarization modes [1]. The latter effect is due to the birefringence splitting that results in different positions of the modes with respect to the gain curve. Thus, the two modes experience a different material gain and index of refraction.

If the injection current is increased, the mode selected at the threshold can become unstable. Different scenarios of polarization instabilities have been described: the state of polarization can switch to the orthogonal polarization $[1,3,5,7]$, random hopping between the two modes may happen at the switching current $[4,6]$ or dynamical states of a highly undefined polarization can occur [7].

There have been several proposals for the mechanisms inducing the polarization instabilities. One class of models is based on the change of the linear anisotropies by thermal effects that come into play via ohmic heating as the current is increased $[1,14,17]$. One important effect of a change in (lattice) temperature is a change of the detuning between the gain peak and the cavity resonances $[1,13]$. This is because the amount of redshift for increasing temperature is different for these two quantities (the redshift of the gain peak is stronger). Since the two polarization modes are frequency split due to birefringence, this shift of the modes on the gain curve results in a change of the linear net gain anisotropy. If this change is sufficiently strong, a polarization switching can occur [1]. In a refined treatment one might add the effect of temperature-dependent losses [17]. While these thermal models have been able to explain some of the experimentally observed switching scenarios and the polarization selection at threshold in dependence of the temperature in most of the investigated VCSEL devices, they cannot explain many experimentally observed dynamical properties, e.g., current dependent nonlinear anisotropies [3] or the dynamical states observed in Ref. [7].

A different approach is based on the dynamics of carrier populations with opposite spin and nonlinear dispersion $[8,9]$. Here, polarization switching has been shown to be the result of a phase instability. This model has been already successfully used to explain some of the dynamical properties observed in experiments and has been extended in Refs. $[11,13]$ with a frequency dependent semiconductor susceptibility [18] in order to take also temperature-dependent detuning effects into account.

The past investigations have been mainly focused on polarization switching (PS) and dynamics at injection levels above the lasing threshold. Many papers state that a "defined" polarization state is selected at the threshold (e.g., 
Ref. [3]). However, the dynamics at and close to the lasing threshold have been only scarcely investigated in detail. In a few experiments $[5,19]$ the excitation of both orthogonal polarization modes at the threshold, followed by the continuous depletion of one mode for increasing current, has been observed. In Ref. [19] this scenario has been shown to disappear for an anisotropic transverse cavity geometry in which the losses for one mode are increased. Data published in Ref. [20] also show an increase of output power in both modes at the threshold. Both modes also display a similar linewidth characteristics. This behavior has been interpreted as a typical threshold behavior of the nonlasing mode and has been termed "anomalous spontaneous emission." However, none of these investigations examined this scenario in deeper detail.

On the theoretical side, in Ref. [9] the existence of a stable two-frequency solution has been found in simulations for zero dichroism, but the dynamical properties have not been examined.

This paper gives a detailed experimental and theoretical study of the polarization dynamics in VCSELs close to the lasing threshold for the case of stimulated emission of both of the orthogonal polarized modes. Also the differences and similarities to the dynamics in other parameter regimes, i.e., selection of one lasing mode at the threshold followed by a PS at increasing current, will be discussed. It will further be demonstrated that for a suitable set of parameters the model developed in Refs. [11,13] is capable of reproducing the complete scenario that is observed in our experiments.

In the following section we describe the experimental setup, and in Sec. III we give an experimental characterization of the scenario of lasing in both modes at the threshold by investigating the time-averaged polarization properties, the temporal dynamics, and their correlation properties. In Sec. IV we will briefly recall the model described in Ref. [13] and show the results of simulations that reproduce the experimental observations. In Sec. V we will discuss our results.

\section{EXPERIMENTAL SETUP}

The experiments have been performed on commercial gain-guided VCSELs (Emcore Corp., Model 8085-2010) operating in the wavelength region around $840-850 \mathrm{~nm}$. We have chosen devices with an $8 \mu \mathrm{m}$-wide aperture in order to ensure operation in the fundamental transverse mode up to twice the threshold current. The VCSELs have been mounted in a temperature controlled copper holder. The substrate temperature of the VCSELs can be changed and stabilized in a range from $6{ }^{\circ} \mathrm{C}$ to $70^{\circ} \mathrm{C}$. The light emitted from the $\mathrm{VC}$ SEL has been collimated using an aspheric antireflection coated lens. After passing through a half-wave plate, the orthogonal polarized components of the VCSEL output are split by a Wollaston prism. For each polarization component the time-averaged output power and the temporal dynamics can be measured by a low bandwidth detector and an avalanche photodiode (APD) of $1.8 \mathrm{GHz}$ bandwidth, respectively. The output of the avalanche photo diode is recorded with a digital oscilloscope with $1 \mathrm{GHz}$ analog bandwidth (on

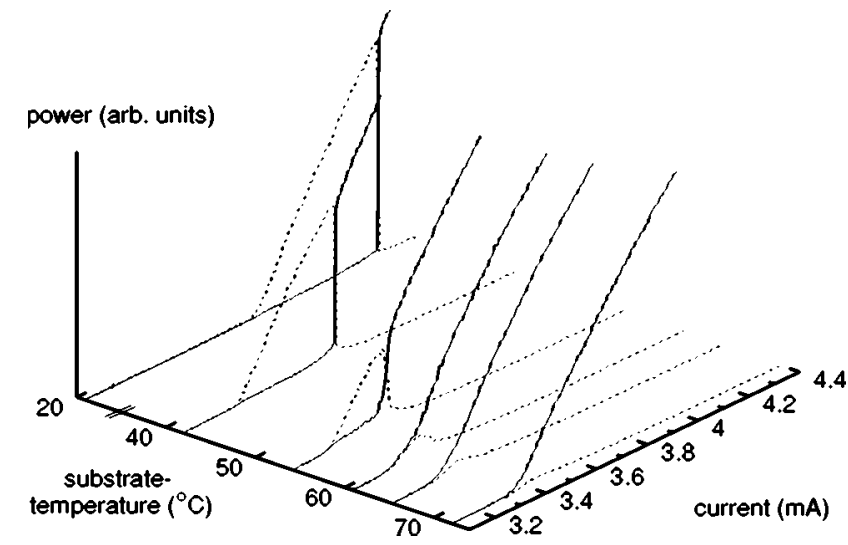

FIG. 1. Polarization resolved power against current ( $L I$ curve) in dependence of the substrate temperature. Solid (dashed) lines denote the power of the mode with lower (higher) optical frequency.

the $5-\mathrm{mV} / \mathrm{div}$ scale). rf spectra are measured with a positiveintrinsic-negative diode of $10 \mathrm{GHz}$ bandwidth and a power spectrum analyzer of $20 \mathrm{GHz}$ bandwidth. A scanning FabryPerot interferometer with a finesse better than 150 and a free spectral range of $46 \mathrm{GHz}$ allows for a measurement of optical spectra. Unintended back reflections into the laser are prevented by an optical isolator.

\section{EXPERIMENTAL RESULTS}

In Fig. 1 the polarization resolved output power of one of the devices under study is displayed in dependence of the injection current and the substrate temperature of the device. At about room temperature, the lasing emission at the threshold is only in the polarization direction corresponding to the mode with higher optical frequency, i.e., the lasing emission is purely linearly polarized. At increasing current, a PS to the mode with lower optical frequency is observed, as it has been often reported in literature. If the substrate temperature of the device is increased, the current value of the PS moves closer to the threshold. With increasing substrate temperature this development continues until finally the point of excitation of the mode with lower optical frequency coincides with the lasing threshold (about $60^{\circ} \mathrm{C}$ ). This leads to emission of both polarizations at the threshold. At increasing current, the mode with higher optical frequency is depleted until only the mode with lower optical frequency is lasing. If the substrate temperature is increased even further, the emission at the threshold is dominated by the mode with lower optical frequency.

This trend closely follows the temperature dependence of the dichroism at the threshold. The value of the dichroism has been obtained with a method explained in Ref. [3,21], i.e., by measurements of rf spectra after projection onto circular polarized states by means of a quarter wave plate. In the regime of excitation of both polarizations at the threshold, the absolute value of the dichroism is of the order of 0.1 $\mathrm{GHz}$ or smaller. We want to stress on the fact that the emission of both polarization components is observed already for sufficiently small but finite dichroism, i.e., for a finite range of substrate temperatures. 


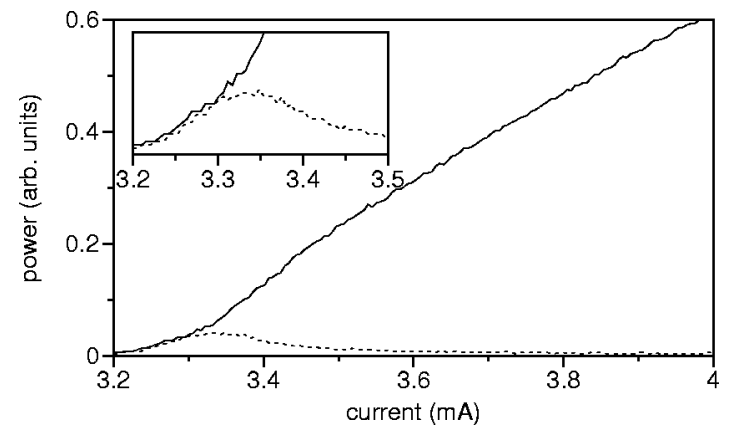

FIG. 2. Polarization resolved, time-averaged power at a substrate temperature of $61{ }^{\circ} \mathrm{C}$. Solid (dashed) lines denote the power of the polarization mode with lower (higher) optical frequency. The inset shows a magnification of the current interval around threshold.

In subsequent realizations of the experiment the center of the region with two-frequency emission was found to fluctuate by a few degrees. Some fluctuations in polarization behavior of VCSEL devices were reported before $[3,22]$. The origin of these fluctuations is not completely clear but is probably related to minute changes of the stress and strain characteristic exercised by the mounting on the VCSEL $[5,15,23]$.

Figure 2 shows the polarization resolved power as a function of the injection current ( $L I$ curve) with the substrate temperature set to the regime in which the laser emits in both modes with equal power at threshold $\left(61{ }^{\circ} \mathrm{C}\right)$ in greater detail. At threshold, both of the orthogonal polarization modes start to lase with equal time-averaged power. Up to $\approx 4 \%$ above the threshold the power increases equally for both modes. Then a preference of the mode with lower optical frequency is observed. The power in the mode with higher optical frequency still increases, until it reaches a maximum at slightly higher current, values. For further increasing current the power in this mode continuously decreases until it reaches the spontaneous emission level. The optical spectrum at the threshold shows two peaks of equal magnitude with orthogonal linear polarizations corresponding to the two modes, i.e., the presence of power in both polarization directions cannot be attributed to a single elliptically polarized lasing mode. In the following, we will call the current interval in which both modes are lasing two-frequency emission (TFE) regime.

Further, the rf spectra after projection on the polarization directions of the two modes have been examined. For both modes, relaxation oscillation (RO) peaks are observed in the vicinity of the lasing threshold. This is a confirmation that both modes are lasing. After depletion of the mode with higher optical frequency to the spontaneous emission level, a RO peak is not observed anymore in the rf spectra of this mode.

Figure 3 displays the temporal dynamics at constant current in the region of TFE. Part (a) shows the dynamics at the lasing threshold. In both polarization directions bursts starting from the spontaneous emission level are observed. The bursts have amplitudes of an equal order of magnitude for both polarization components and appear with the same probability in a fixed time interval. This corresponds to the
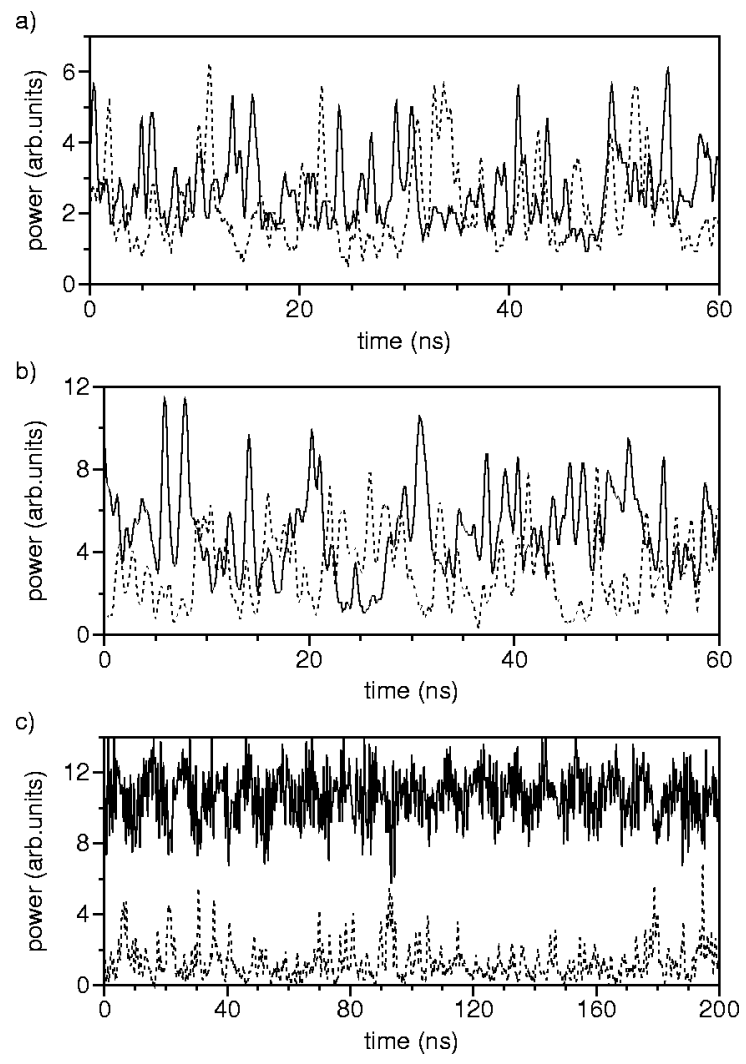

FIG. 3. Polarization resolved time traces in the regime of twofrequency emission: $1 \%$ above the lasing threshold (a), at the point of maximum power of the mode with higher optical frequency $(4 \%$ above the threshold) (b), and $8 \%$ above the threshold (c). Solid (dashed) lines denote the power of the polarization mode with lower (higher) optical frequency (bandwidth $1 \mathrm{GHz}$ ).

fact that the time-averaged power was observed to be equal for both polarization modes. Up to $4 \%$ above the threshold, the dynamics do not change qualitatively [Fig. 3(b)]. The amplitude of the fluctuations in both components is of the same order of magnitude as the average power. If the current is increased beyond $4 \%$ above the threshold value, the bursts in the polarization mode with higher optical frequency appear less frequently than the ones in the other mode and their amplitude decreases [Fig. 3(c)]. In the whole regime of emission of both polarization modes in the vicinity of the lasing threshold, mode hopping (i.e., square-wave-like signals due to the abrupt alternation between the two polarizations states) as reported in literature for PS higher above the threshold (e.g., Ref. [4]) is not observed.

In Fig. 4 the correlation properties of the time series displayed in Fig. 3(b) are shown. The correlation functions have been calculated from the time traces by use of the WienerKhintchine theorem. The autocorrelation functions of both modes exhibit a distinct modulation with the frequency of the modulation being the same for both modes. This modulation is also present in the cross correlation function of the dynamics. Further, the cross correlation function reveals a clear anticorrelation of the dynamics in the two polarization directions and is slightly asymmetric with respect to zero 


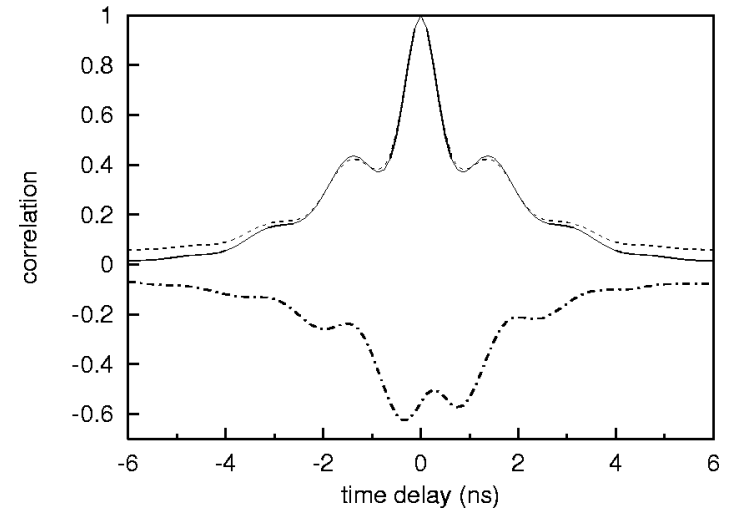

FIG. 4. Correlation properties of the dynamics displayed in Fig. 3(b): the solid (dashed) line displays the autocorrelation function of the mode with lower (higher) optical frequency, the dash-dotted line represents the cross correlation function of the two modes.

time lag. The (anti)correlation decays to zero within a few nanoseconds.

The minimum of the cross correlation functions in dependence on the injection current is displayed in Fig. 5. The modulus of the anticorrelation is continuously increasing in the current interval where both modes lase with equal power (see Fig. 2). When the power of the mode with lower optical frequency surpasses the power in the orthogonally polarized mode, the modulus of the anticorrelation reaches a maximum and decreases afterwards.

To identify the origin of the modulation in the cross correlation functions (see Fig. 4), we have plotted in Fig. 6 the square of the frequency of this modulation as a function of current. It has an approximate linear dependence on current which is characteristic for relaxation oscillations (e.g., Ref. [24]). For comparison, the square of the RO frequencies measured in the spectral domain is also plotted in Fig. 6. Within some fluctuations, the modulations of the cross correlation functions agree with the RO frequencies and show the same characteristic dependence on current. Hence, it can be concluded that the modulation appearing in the correlation functions in Fig. 4 is due to the ROs.

To clarify the influence of the different frequency components on the dynamics, we have computed the normalized cross spectral density (NCSD) of the two time traces of Fig. 3 (b). The NCSD is obtained from the two time traces $I_{x, y}(t)$ by Fourier transformation (denoted in the formula below by a tilde) and use of the relationship

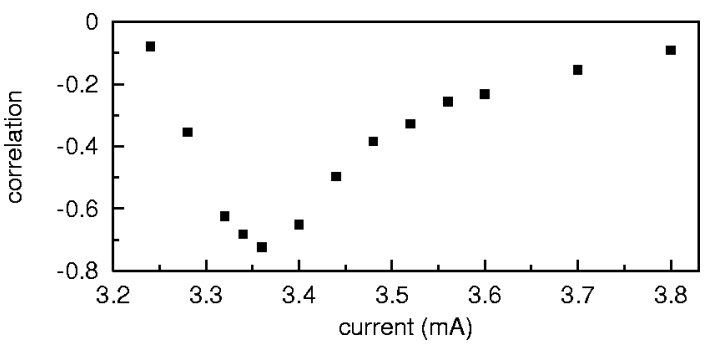

FIG. 5. Minimum of the cross correlation function of the temporal dynamics of the two polarization modes in dependence of the injection current.

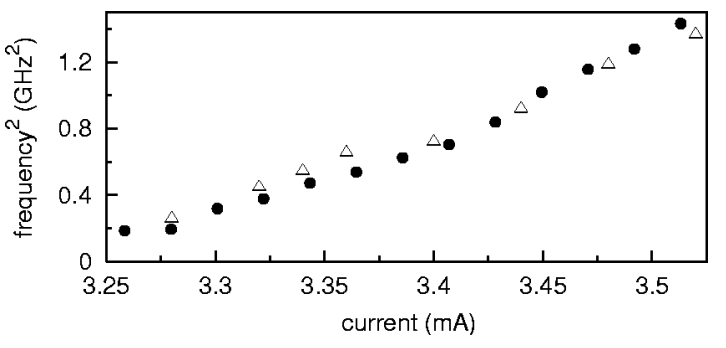

FIG. 6. Square of the frequency of the relaxation oscillations (triangles) and of the modulation of the cross correlation function (circles) of the time series of the polarization modes in dependence of the injection current.

$$
C(f)=\frac{\tilde{I}_{x}(f) \tilde{I}_{y}^{*}(f)}{\sqrt{\left|\tilde{I}_{x}(f)\right|^{2}\left|\tilde{I}_{y}(f)\right|^{2}}},
$$

where for each frequency of modulus $f$ the contributions from frequencies $f$ and $-f$ have been added. This results in a real number for $C(f)$. The NCSD contains information about the amount of (anti)correlation at a certain frequency. The results are given in Fig. 7. At low frequencies, the dynamics are almost perfectly anticorrelated (the features observed for frequencies less than $150 \mathrm{MHz}$ are due to broadcast radio signals captured by the APDs and they appear even when the output of the VCSEL is blocked). This corresponds to the overall anticorrelation at zero time lag in the cross correlation function in the time domain (see Fig. 4) and the rather slow decay of the anticorrelation for larger time lags. Anticorrelation at low frequencies has been shown to be a robust feature of the polarization dynamics of VCSELs in past investigations $[25,26]$. For increasing frequencies, the correlation increases (i.e., the modulus of the anticorrelation decreases) until the NCSD reaches a maximum at $0.6 \mathrm{GHz}$ with a normalized correlation value of about 0.3 . At further increasing frequency, the NCSD decays towards zero. The frequency of the maximum corresponds to the RO frequency of the total power. Since the RO is a process that acts on the

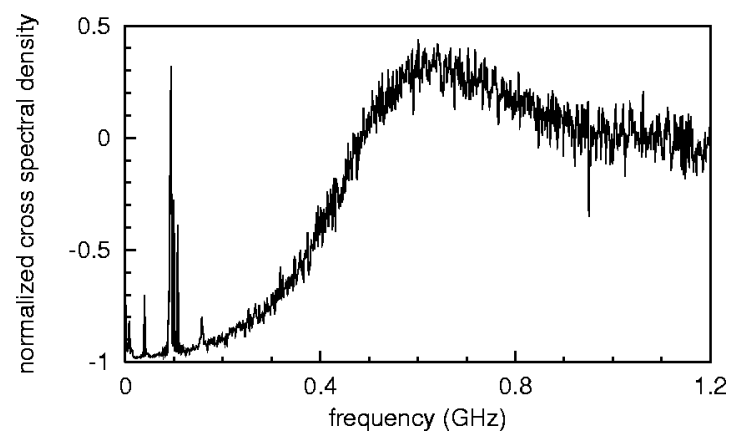

FIG. 7. Normalized cross spectral density of the two time traces displayed in Fig. 3(b). The fluctuations visible for frequencies less than $150 \mathrm{MHz}$ are induced by perturbations due to broadcast radio signals. They have been checked to enter into the APDs and to appear also if the output of the VCSEL is blocked. 

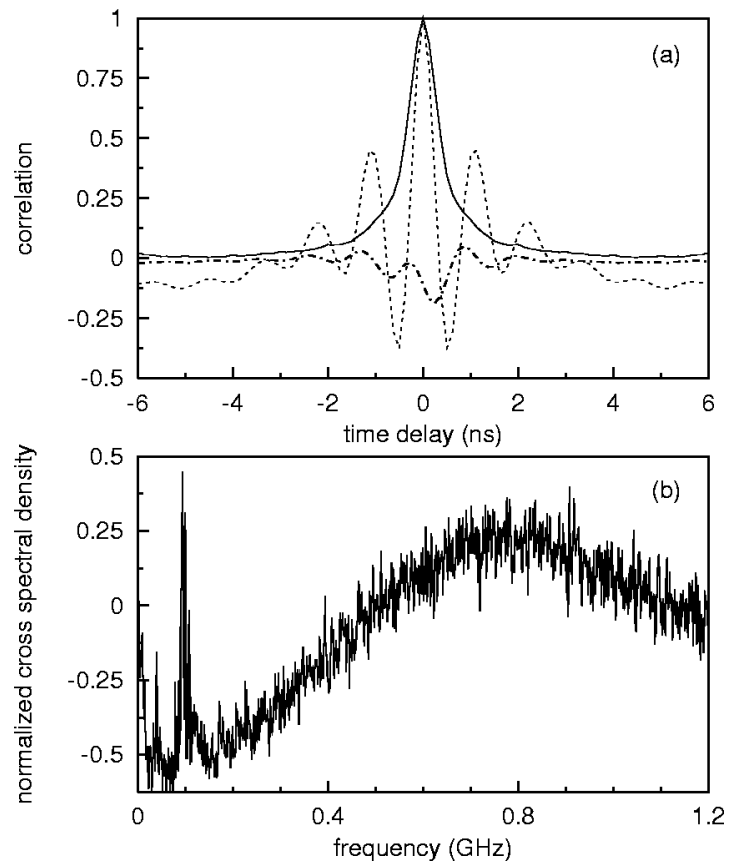

FIG. 8. Correlation properties of the dynamics at $21^{\circ} \mathrm{C}$ and $4 \%$ above the lasing threshold: correlation functions in the time domain (a) and normalized cross spectral density (b). The lines in (a) denote the same properties as in Fig. 4.

total inversion and both polarization modes are lasing in the regime under study, they have to be influenced simultaneously.

This explains why the anticorrelation shown in Fig. 5 is not complete (i.e., -1 ) although the NCSD at low frequencies is perfect: at zero time lag we have a contribution from all the frequency components of the NCSD, the highfrequency components reducing the anticorrelation due to the low-frequency ones.

For comparison, we finally analyze the correlation properties of the dynamics of the polarization modes in the regime where only one linearly polarized mode is selected at the threshold and PS occurs, if the current is increased. The correlation properties of this regime are displayed in Fig. 8 for a substrate temperature of $21^{\circ} \mathrm{C}$ (see leftmost $L I$ curve in Fig. 1), and for a better comparison with the dynamics in the two-frequency emission regime a current level of $4 \%$ above the threshold has been chosen. As expected, in the autocorrelation function of the lasing mode, which is now the mode with higher optical frequency, a modulation at the RO frequency is observed. In the autocorrelation function of the nonlasing mode, this modulation is almost not detectable. Correspondingly, only the power spectrum of the lasing mode exhibits a measurable RO peak (not shown). In the time domain, only few bursts that originate from the noise level are observed for the nonlasing mode. The dynamics in this regime is comparable to the dynamics in the regime of TFE far above the threshold, i.e., where the major fraction of the total power is emitted in one mode. Nevertheless, a modulation at the RO frequency is also observed in the cross correlation function. Correspondingly, the NCSD shows weak correlation $(\leqslant 0.25)$ about the RO frequency of 0.8
GHz. The amount of anticorrelation is decreased with respect to the TFE regime. In the time domain, the minimum of the cross correlation function is about -0.17 . In accordance with this lower value, the anticorrelation at low frequencies in the NCSD is about -0.5 , i.e., the modulus of the anticorrelation at low frequencies has drastically decreased with respect to the TFE regime. To summarize the above findings, also in the regime of selection of only one lasing mode at the threshold the dynamics close to the threshold are characterized by correlated fluctuations at the RO frequency. The magnitude of the correlation at this frequency is approximately the same as in the TFE regime, whereas the amount of anticorrelation at low frequencies is significantly decreased.

If the current is now increased to the point of PS, the dynamics at low frequencies again become almost completely anticorrelated. The NCSD at low frequencies is only slightly larger than -1 , as it has been reported for PS also in Ref. [25]. In the time domain, mode hopping of the two polarization modes at the PS is observed, as it has also been reported in the literature before $[4,6,27]$. Whether a correlation at the RO frequency can still be found is unclear from an experimental point of view, since the RO frequency has left the bandwidth of the oscilloscope at the current of the PS.

\section{THEORY}

In this section we theoretically investigate the polarization dynamics occurring within the TFE regime. In the model discussed in this section, polarization mode selection occurs through a combination of several mechanisms, namely, spinflip processes, saturable dispersion, and thermal shift $[11,13]$. The latter is essential since we need to describe the dynamics in a finite range of detunings in order to reproduce the results discussed in the experimental section (Fig. 1).

Under fundamental transverse mode operation, the evolution of the circularly polarized components of the electric field $E_{ \pm}$(slowly varying envelopes) and the electronic densities $D_{ \pm}$with opposite spin (normalized to the transparency density) are governed by $[11,13,28,29]$

$$
\begin{aligned}
\dot{E}_{ \pm}(t)= & -\kappa E_{ \pm}+i \frac{a \Gamma}{2} \chi_{ \pm}\left(\Omega+i \frac{\dot{E}_{ \pm}}{E_{ \pm}}, D_{+}, D_{-}\right) E_{ \pm} \\
& -\left(\gamma_{a}+i \gamma_{p}\right) E_{\mp}+\sqrt{\beta_{s p} D_{ \pm}} \xi_{ \pm}(t), \\
\dot{D}_{ \pm}(t)= & \frac{\mu}{2}-A D_{ \pm}-B D_{ \pm}^{2} \mp \gamma_{j}\left(D_{+}-D_{-}\right) \\
& +a \operatorname{Im} \chi_{ \pm}\left(\Omega+i \frac{\dot{E}_{ \pm}}{E_{ \pm}}, D_{+}, D_{-}\right)\left|E_{ \pm}\right|^{2} .
\end{aligned}
$$

The electronic densities with opposite spin interact with circularly polarized light with different helicity through the frequency dependent susceptibility $\chi_{ \pm}[11,30]$. We use analytical expressions for $\chi_{ \pm}$obtained in Ref. [18] that correctly describe the gain and refractive index spectra. Spin-flip processes, that reverse the electron spin, directly couple the two carrier reservoirs. This effect is phenomenologically ac- 


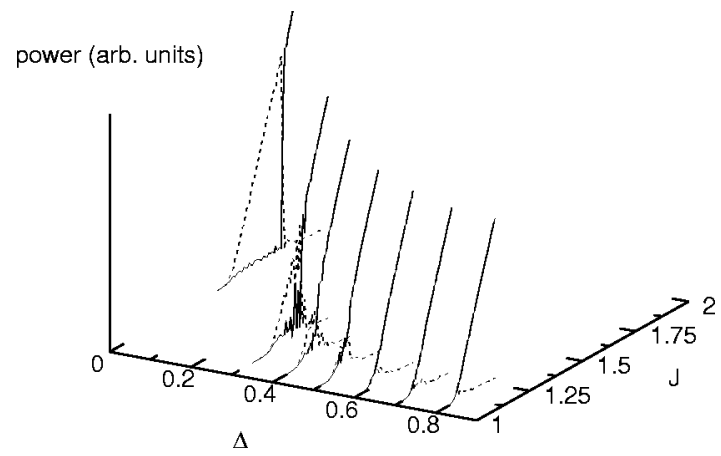

FIG. 9. Polarization resolved output power in dependence of the detuning $\Delta$ and injection current $J$ normalized to its transparency value. The lines denote the same properties as in Fig. 1. The parameters defining the susceptibility function [18] are $b=1 \times 10^{4}, \gamma$ $=1 \times 10^{4} \mathrm{~ns}^{-1}$, and $\sigma=0.2$.

counted for by means of the spin-flip rate $\gamma_{j}$. The thermal shift is incorporated through the temperature dependence of the detuning $\Delta$ between the cavity resonance and the nominal transition frequency. $\mu$ is the total injected current density scaled to the transparency carrier density.

The parameters of the above equations are taken from the experimental conditions: the linear contribution to the birefringence and dichroism $\gamma_{p}=18 \mathrm{~ns}^{-1}$ and $\gamma_{a} \approx 0$. To obtain the frequency splitting one has to divide $\gamma_{p}$ by $\pi$. In the framework of this model $\gamma_{a}$ is a pure loss anisotropy. Material gain differences are incorporated by the optical susceptibility $\chi_{ \pm}$. The meaning and values of the rest of parameters are the cavity losses $\kappa=300 \mathrm{~ns}^{-1}$, the effective gain constant $a=2.3 \times 10^{4} \mathrm{~ns}^{-1}$, the confinement factor $\Gamma$ $=0.045$, the nonradiative and bimolecular recombination rates of the carriers $A=0.5 \mathrm{~ns}^{-1}$ and $B=1.0 \mathrm{~ns}^{-1}$, and the spontaneous emission rate $\beta_{s p}=10^{-6} \mathrm{~ns}^{-1}$. The values assumed for the parameters $\kappa, A, B$ are considered as typical values for VCSEL devices. One could use them in order to fine tune the absolute magnitude of the RO. We did not try that since we are only interested in the robust features of the polarization dynamics.

In most of the simulations a value of $\gamma_{j}=20 \mathrm{~ns}^{-1}$ was chosen. A discussion of the influence of the magnitude of the spin-flip rate will be given at the end of this section. Finally, $\xi_{ \pm}(t)$ are white Gaussian random numbers with zero mean and $\delta$ correlated in time that model spontaneous emission processes.

The simulations have been performed in the circular polarized basis. To obtain expressions for linear polarized fields one has to use the relations $E_{x}=\left(E_{+}+E_{-}\right) / \sqrt{2}, E_{y}=\left(E_{+}\right.$ $\left.-E_{-}\right) /(i \sqrt{2})$.

As a first step, $L I$ curves for various detunings have been simulated (see Fig. 9). By a change of the detuning a variation of substrate temperature as performed in the experiments can be simulated. The current has been increased in 50 steps from $5 \%$ below to $20 \%$ above the threshold within $5 \mu \mathrm{s}$. As it is obvious from Fig. 9, the simulations reproduce the experimentally measured polarization properties qualitatively for variation of both the detuning and the current.

A regime of TFE is found in the simulations in the vicin-

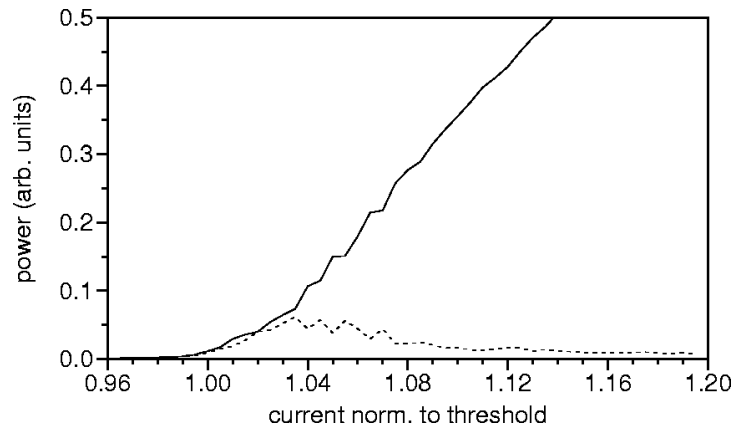

FIG. 10. Simulated $L I$ curve for $\Delta=0.65$. The integration time is $100 \mathrm{~ns}$ per current value.

ity of the threshold minimum, which occurs at a detuning of $\Delta=0.65$. At this detuning value, a linear stability analysis (LSA) of the two orthogonally linear polarized modes (see Ref. [11]) yields bistability at the threshold between the two modes, both of which remain stable upon increasing the current up to twice the threshold current. Within a finite interval of detuning (about $0.6 \leqslant \Delta \leqslant 0.8$ ), both polarizations are excited at the threshold in the simulations including noise, although only one mode is linearly stable directly at the threshold for $\Delta \neq 0.65$. (However, within the interval mentioned above the other mode reaches stability if the current is slightly increased.) Therefore, as in the experiment, the TFE regime extends over a finite interval of detuning. Outside this
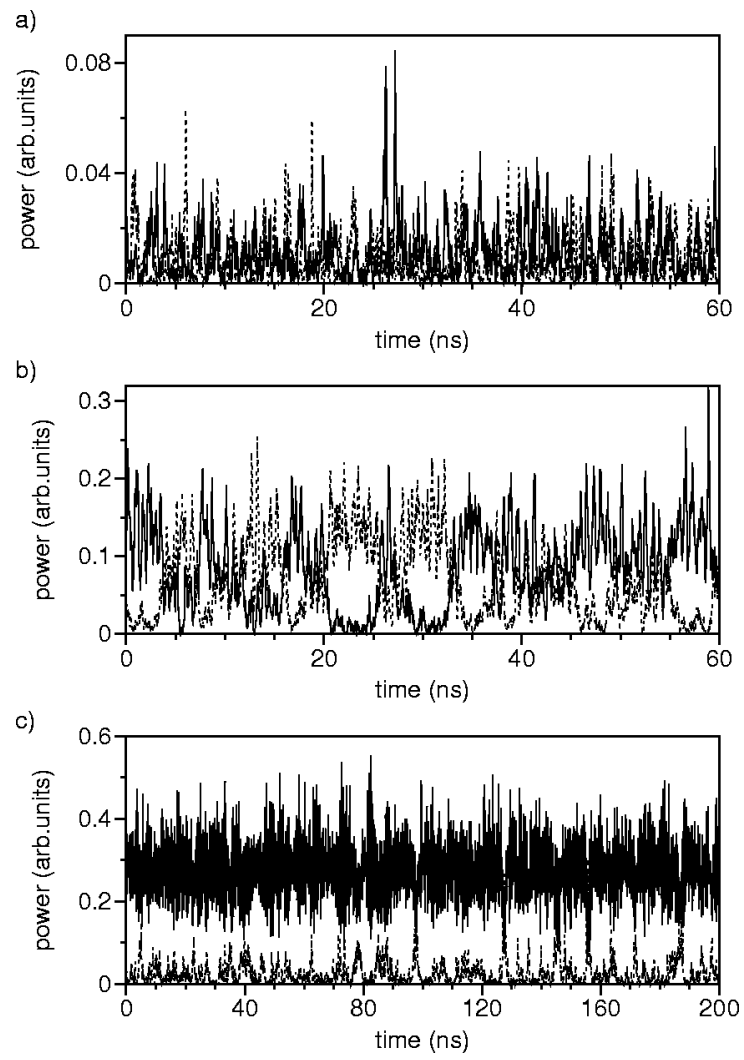

FIG. 11. Simulated polarization resolved time traces at $\Delta$ $=0.65$ : at threshold (a), $4 \%$ above threshold (b), and $8 \%$ above threshold (c). Solid (dashed) lines denote the power of the polarization mode with lower (higher) optical frequency. 

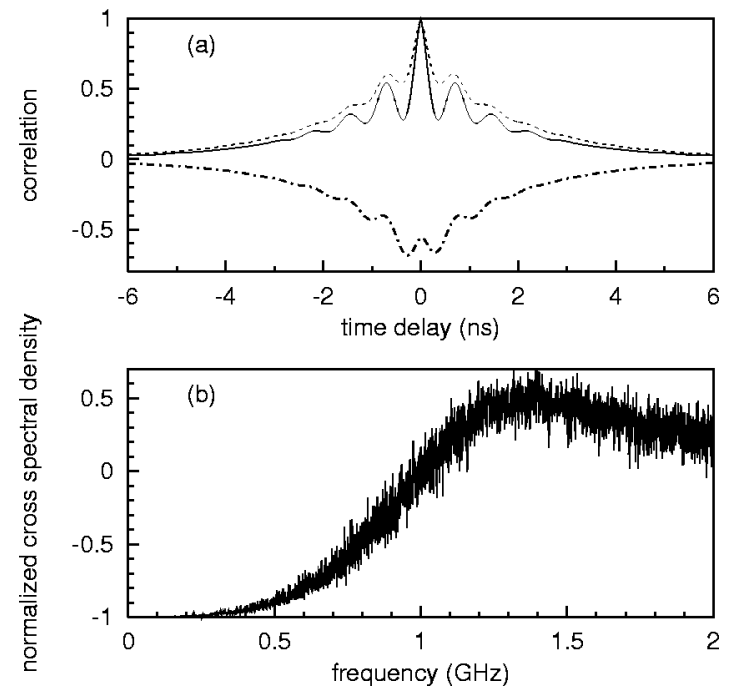

FIG. 12. Correlation properties of the dynamics displayed in Fig. 11(b): correlation functions in the time domain (a) and normalized cross spectral density (b). The lines in (a) denote the same properties as in Fig. 4. The length of the analyzed time traces is $50 \mu \mathrm{s}$, which matches the length of the experimental traces.

interval, the low-frequency mode is dominant at the threshold for higher detuning values, the high-frequency mode otherwise. For $\Delta=0.65$ the $L I$ curve is displayed in greater detail in Fig. 10. The qualitative features are the same as in the experiment: Both modes emit with equally increasing power close to the threshold. At a current level of $4 \%$ the mode with higher optical frequency reaches its maximum value and decays continuously afterwards.

Examples of the temporal dynamics in the TFE regime are shown in Fig. 11. The dynamics resemble the experimentally obtained results, i.e., they are dominated by burstlike fluctuations. For increasing current, the probability and amplitude of a burst in the mode with higher optical frequency decreases continuously.

The correlation properties of the dynamics in the TFE in the simulations (Fig. 12) agree with the experimentally obtained ones: The dynamics are anticorrelated at low frequencies and correlated at the RO frequency (about $1.4 \mathrm{GHz}$ for the parameters in Fig. 12). The minimum value of the cross correlation function $[-0.7$, see Fig. $12(\mathrm{a})]$ is rather close to the experimentally obtained one $(-0.64)$.

Also the current dependence of the minimum of the cross correlation function shows the same characteristics observed in the experiments (see Fig. 13), i.e., the anticorrelation is strongest when the power in the mode with higher optical frequency is at its maximum value. However, the minimum is somehow broader (plateaulike) in the simulations.

If the detuning $\Delta$ is decreased, a continuous transition from TFE to the selection of the mode with higher frequency at the threshold and PS at increasing current is observed (see Fig. 9), i.e., the current value of maximum polarization mode competition moves to higher currents at decreasing detuning. The dynamics and the corresponding correlation properties at low detuning values qualitatively reproduce the experimental ones obtained for low substrate temperatures, i.e., also close

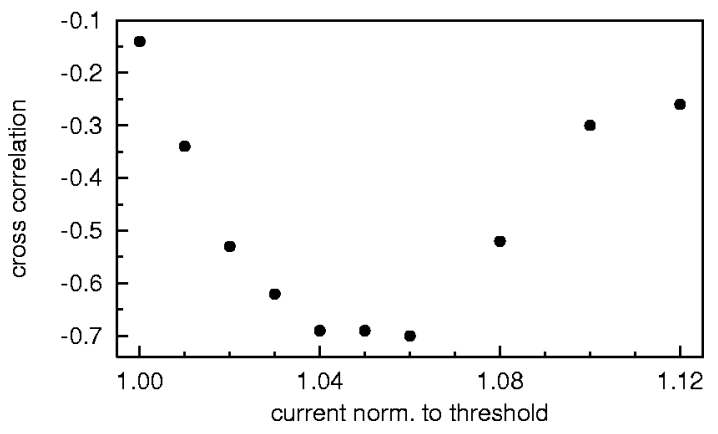

FIG. 13. Same as Fig. 5, but for simulated time traces at $\Delta$ $=0.65$.

above the threshold the cross correlation function in the time domain yields anticorrelation and is modulated at the RO frequency although only one mode is lasing.

If the current is increased to the point of PS, mode hopping is observed. As in earlier publications (e.g., Ref. [25]), the cross correlation at low frequencies is close to -1 [see Fig. 14(b)]. However, the minimum correlation found in the temporal cross correlation function [Fig. 14(a)] is $\sim-0.9$. This is due to the fact that slightly correlated dynamics occur around the RO frequency $(2.3 \mathrm{GHz}$ in this case). Consequently, also in this case a modulation at the RO frequency is observed in the cross correlation function [see inset in Fig. 14(a)].

With the set of parameters used, we obtain a good quantitative agreement with the experimental observations. If $\gamma_{a}$ is not zero but still small, the point at which the two polarization modes exchange stability at the threshold shifts away from the threshold minimum (see also Refs. [11,13]). The direction of the shift depends on the sign of $\gamma_{a}$. In the vicinity of this point there is still a two-frequency emission regime with the characteristic dynamics reported above. As reported in the preceding section, equivalent scenarios have been observed in the experiments.
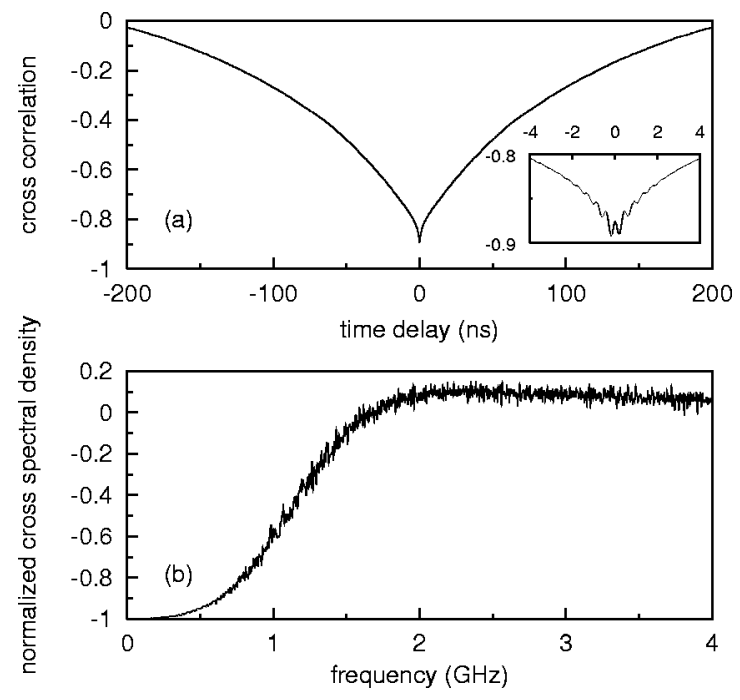

FIG. 14. Correlation properties of the dynamics at a PS occurring at $\Delta=0$ and $17 \%$ above threshold: cross correlation function in the time domain (a) and normalized cross spectral density (b). The length of the analyzed time traces is $50 \mu \mathrm{s}$. 


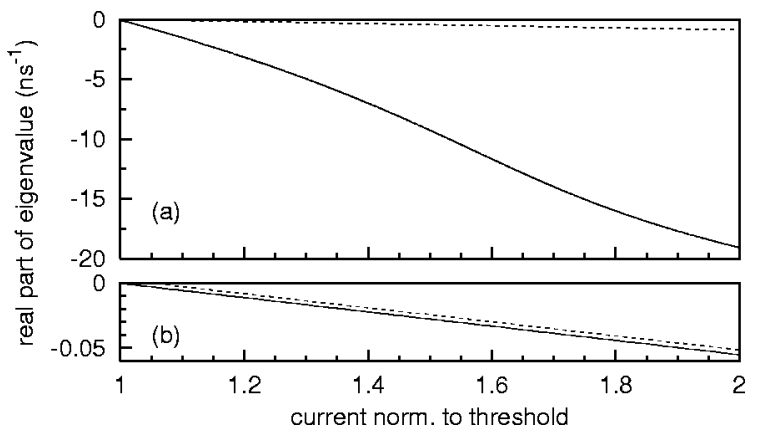

FIG. 15. Real part of the complex conjugate eigenvalues obtained from an analysis of the linear stability of the lasing solutions against orthogonally polarized perturbations for parameters as in Fig. 10 and $\gamma_{j}=20 \mathrm{~ns}^{-1}$ (a), $\gamma_{j}=5000 \mathrm{~ns}^{-1}$ (b). Solid (dashed) lines denote the eigenvalue describing the stability of the mode with lower (higher) optical frequency.

A variation of the noise strength does not change the dynamics in the TFE regime qualitatively. If $\beta_{s p}$ is decreased (increased), the current value of maximum output power and the maximum power itself of the mode with higherfrequency decrease (increase). The fact that the mode with higher optical frequency is depleted in a continuous way for increasing current is unchanged.

An interesting question regarding the TFE is why one mode is depleted at increasing current. Indeed, the LSA of the pure linearly polarized modes predicts that in this current range both modes are still bistable, and they remain so up to much higher current values. Hence one would expect to observe them all over the range of bistability due to noise. However, the stability of each of these pure linearly polarized modes is given by the eigenvalue with the smallest real part in the LSA. For each pure linearly polarized state, the LSA yields three eigenvalues, of which one is real and the others form a complex conjugate pair (having the same real part, of course). The real parts of these eigenvalues describe the exponential decay or growth of a perturbation in time, depending on the sign of the real part. In the parameter region considered here, the real eigenvalue is negative and of larger modulus than the real part of the complex eigenvalues. Therefore it can be neglected in the following discussion. Figure 15(a) displays the results of the LSA of the lasing solutions against perturbations with orthogonal polarization represented by the complex eigenvalues. As it is apparent from Fig. 15(a), both modes are stable up to $200 \%$ above the threshold, i.e., the real parts of the eigenvalues of both modes are negative. However, the modulus of the eigenvalue describing the stability of the mode with lower frequency increases stronger with current and is about an order of magnitude larger, already close above the threshold. This implies that perturbations to the mode with lower frequency are damped an order of magnitude faster. Thus, one can call this mode "more stable." As observed in the simulations, the system "chooses" this mode and the other mode is depleted with increasing current or increasing difference in stability, respectively, although the linear (unsaturated) net gain anisotropy does not change with current.

To study the influence of the spin dynamics, we have
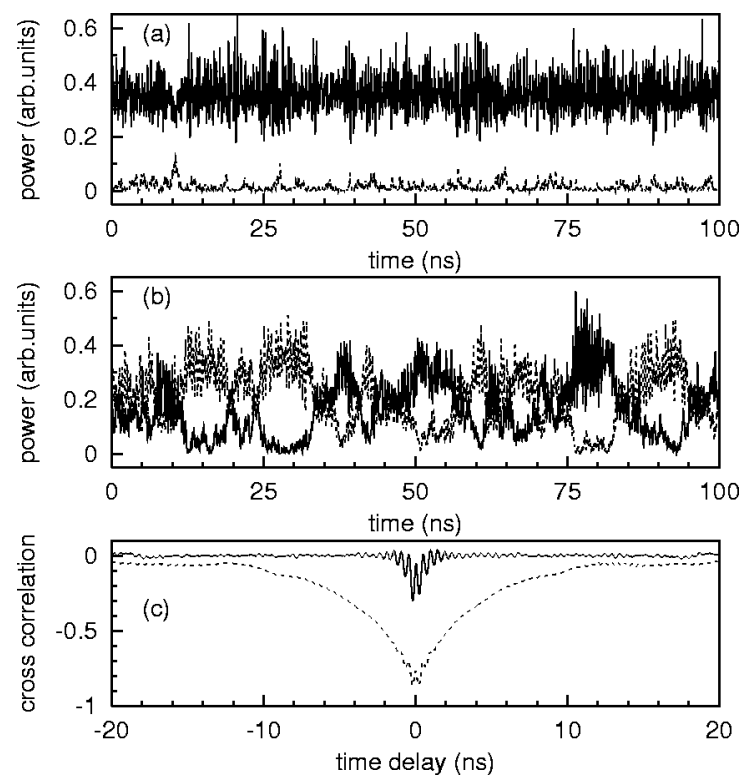

FIG. 16. Polarization dynamics at $\Delta=0.65$ and $10 \%$ above the threshold for $\gamma_{j}=20 \mathrm{~ns}^{-1}$ (a), and $\gamma_{j}=5000 \mathrm{~ns}^{-1}$ (b). Solid (dashed) lines denote the power of the mode with lower (higher) optical frequency. The corresponding cross correlation functions are displayed in (c), where a solid and dashed line represent the correlation function of the dynamics in (a) and (b), respectively.

repeated the LSA for a large value of the spin-flip rate $\left(\gamma_{j}\right.$ $=5000 \mathrm{~ns}^{-1}$ ). This is practically equivalent to a neglect of spin dynamics in the model discussed here (assuming $D_{+}$ $=D_{-}$, i.e., $\gamma_{j} \rightarrow \infty$ as in Refs. [31,32]). The corresponding results are given in Fig. 15(b). The bistability of the two polarization modes is maintained. However, the modulus of the real parts of the eigenvalues is now decreased by two orders of magnitude, i.e., perturbations are generally less damped. Furthermore, the real parts and thus the damping of a perturbation are approximately the same for both polarization modes. In a spin eliminated version of the original spinflip model (e.g., Ref. [10]) exact equality for both damping values is analytically predicted. Consequently, the two modes should be equivalent and none of them should be depleted at increasing current in the long-time limit.

The latter assumption has been tested for a current level of $10 \%$ above the threshold (Fig. 16). For a small spin relaxation rate, the mode with higher optical frequency is almost completely depleted [see Fig. 16(a) and also the $L I$ curve in Fig. 10]. The cross correlation function exhibits a rather small amount of anticorrelation $(\sim-0.3)$ at zero time delay and a pronounced modulation at the RO frequency [Fig 16(c)]. For $\gamma_{j}=5000 \mathrm{~ns}^{-1}$ the dynamics change dramatically and strong mode competition between the two polarization modes is observed [Fig. 16(b)]. The mode with higher frequency is not depleted anymore within the investigated current range, which goes up to three times the threshold current. The corresponding cross correlation function now exhibits a strong anticorrelation $(-0.85)$, which is due to the completely anticorrelated dynamics at low frequencies. Furthermore, the asymmetry of the cross correlation function that is observed for the lower spin-flip rate is lost because 
both modes now participate equally in the dynamics. The correlation properties obtained here are similar to the ones observed at the PS at low detuning and for small spin-flip rates (see Fig. 14). This is due to the fact that also in the latter case the real parts of the eigenvalues of the two modes differ only slightly [33].

Furthermore, for sufficiently high $\gamma_{j}$ the transition to PS with decreasing detuning is not observed anymore. The mode that is selected as the lasing mode at the threshold (depending on $\Delta$ ) remains lasing at increasing current, whereas the other mode remains off at all investigated current values.

For values of the spin-flip rate between the two cases discussed above, the dynamics in the TFE regime change continuously. Starting with $\gamma_{j}=20 \mathrm{~ns}^{-1}$, an increase of $\gamma_{j}$ leads to an increase of the maximum power of the mode with higher frequency and the corresponding current value, until the scenario described above is obtained.

\section{DISCUSSION}

The experimental and theoretical investigations have shown that the polarization dynamics in the two-frequency emission regime at the threshold is strongly influenced by the $\mathrm{RO}$, which are known to be only weakly damped close to the threshold. The dynamics was shown to be not completely anticorrelated, but rather have correlated components at the RO frequency, which induce a decrease of the degree of anticorrelation in the time domain. Since the RO is a process acting on the total power, it can be expected that the dynamics at the RO frequency are correlated. In an earlier publication [26] it was shown that the correlation at low frequencies depends on the distribution of power between the two modes, i.e., the modulus of the anticorrelation increases towards one if the power in both modes approaches equality. The latter observation agrees well with the current dependence of the anticorrelation in the TFE regime, as it was found here.

In previous investigations, the competition between bistable polarization states in the vicinity of a PS has been often found to be accompanied by completely anticorrelated stochastic hopping between the two modes yielding a sequence of square-like or rectangular-like pulses in the polarization resolved time series $[4,6]$. This is evidently neither the case in the experimental (Fig. 3) nor in the simulated (Fig. 11) time series considered here. This might be related to the fact that in the TFE regime the fluctuations in power, namely, the ROs, are of the same magnitude as the average power. Furthermore, at the threshold the respective time scales become different from the ones considered in the situations before. Higher above the threshold, the RO period is typically less than a nanosecond and the dwell times found have not been smaller than $10 \mathrm{~ns}[4,6]$ (the lowest reported values stem from currents $20 \%$ above the threshold). For decreasing current, i.e., smaller distance to the threshold, the RO period increases and the dwell-time decreases. Hence, both time scales can become of similar magnitude close to the threshold. Since the dynamics are correlated at the RO frequency, the system is not likely to be found in only one of the orthogonal polarizations during one $\mathrm{RO}$ cycle.

The scenarios depicted in Figs. 1 and 9 suggest that twofrequency emission occurs in the vicinity of a zero crossing of the linear dichroism at the threshold in dependence of detuning or substrate temperature, respectively. Whereas in a deterministic analysis $[11,13]$ polarization selection is perfect at the threshold in the moment in which the dichroism deviates only slightly from zero, this is not the case in the presence of spontaneous emission noise, which smears out the nonlasing to lasing transition and continuously feeds all field components. Hence, two-frequency emission is observed in a finite interval of substrate temperature (detuning) in which none of the modes is strongly favored.

For low values of the spin-flip rate, one of the polarization components, which is selected at the threshold for a sufficiently high value of the dichroism, is destabilized again at higher currents and polarization switching to the orthogonal polarization state occurs (Fig. 11, see also [11,13]). The PS and the accompanying bistable region shift continuously away from the threshold, if the temperature (detuning parameter) is reduced. In this sense, two-frequency emission might be regarded as the manifestation of bistability between two polarization components at and close to the laser threshold. However, as discussed above, the polarization dynamics in this regime do not show a finite dwell time as in previous experiments, due to the proximity to the threshold for laser emission.

The experiments and the simulations show very good agreement if the spin-flip rate is assumed to be low. Pumpprobe experiments on passive quantum film structures yielded even lower values of $\gamma_{j} \approx 7-9 \times 10^{9} \mathrm{~s}^{-1}$ at room temperature $[34,35]$. However, it is doubtful whether these values provide a good estimation for the situation of a high carrier density typical for laser operating conditions. Previous estimations of the spin-flip rate under lasing conditions were inferred indirectly from experiments assuming the validity of the spin-flip model (SFM) and yielded $\gamma_{j} \approx 30-75$ $\times 10^{9} \mathrm{~s}^{-1}$ [36], $\gamma_{j}>100 \times 10^{9} \mathrm{~s}^{-1}$ [3], $\gamma_{j} \approx 10^{12} \mathrm{~s}^{-1}$ [37], and "infinity" [31], i.e., the values reported in the literature span over a rather wide range but are significantly higher, in tendency, than the values considered here. However, the low rate used here is of the same order as needed to explain the self-pulsing [38] experimentally observed in some VCSEL devices of the same type [7] as well as the dependence of the effective birefringence on current measured in Refs. [7,39]. Thus several-though indirect-observations suggest that the spin-flip rate may be low in the devices under study.

It cannot be excluded that also other models, e.g., the one used in Ref. [31] (i.e., the model of Ref. [2] with the spin-flip rate set to infinity) can predict an excitation of both polarization modes with anticorrelated dynamics if the net gain anisotropy is small enough at the threshold. Indeed, excitation of both modes has also been found in the model used here for large spin-flip rates. Even the depletion of the mode with higher frequency at increasing current and/or the asymmetric cross correlation function can presumingly be modeled in phenomenological rate equations (e.g., Ref. [14]), if a suitable current dependence of the linear anisotropies and suitable cross- and self-saturation coefficients are introduced. 
There is no contradiction to the fact that the dynamics is also described by the SFM. Indeed, it was recently shown that for the type-I polarization switching discussed here the SFM can be reduced to rate equations with asymmetric cross coupling coefficients in some limit [40]. However, in our opinion it is the merit of the SFM that it provides a physical basis for these coefficients and for their parameter dependencies. Furthermore, it is the strength of the model used for comparison in this paper that it is able to reproduce the steady-state characteristics, the dynamics, and the correlation properties simultaneously that have been found in an experiment for changing current and temperature/detuning, where for the modeling only one fixed set of parameters has been used.

\section{CONCLUSIONS}

To conclude, we have given in this paper a detailed experimental and theoretical investigation of the polarization dynamics in the vicinity of the lasing threshold in a VCSEL. Both of the orthogonal polarization modes are found to be lasing at the threshold for a small net gain anisotropy. If the net gain anisotropy is changed by changing the detuning or the substrate temperature, respectively, the two-frequency emission state continuously transforms into a state with defined polarization at the threshold, followed by a polarization switching at higher currents. The dominating time scale of the polarization dynamics at the threshold is given by the relaxation oscillations. The magnitude of correlation between the two modes has been shown to depend significantly on the injection current. We have found a very good agreement between the experiments and the simulations obtained for low spin-flip rates within a model that combines nonlinear spin dynamics and linear thermal effects.

\section{ACKNOWLEDGMENTS}

M.S., M.W., and T.A. gratefully acknowledge financial support by the Deutsche Forschungsgemeinschaft and the Deutsche Akademische Austauschdienst and thank W. Lange for his support and stimulating discussions. S.B. and J.M. gratefully acknowledge financial support by VISTA HCTMR BFM-2000-1108 and TIC 99-0645-C05-02. We are also grateful to S. Barland and F. Marino for discussions and the help in some measurements.
[1] K.D. Choquette, D.A. Richie, and R.E. Leibenguth, Appl. Phys. Lett. 64, 2062 (1994).

[2] J. Martín-Regalado, J.L.A. Chilla, J.J. Rocca, and P. Brusenbach, Appl. Phys. Lett. 70, 3350 (1997).

[3] M.P.v. Exter, M.B. Willemsen, and J.P. Woerdman, Phys. Rev. A 58, 4191 (1998).

[4] M.B. Willemsen, M.U.F. Khalid, M.P.v. Exter, and J.P. Woerdman, Phys. Rev. Lett. 82, 4815 (1999).

[5] K. Panajotov, B. Nagler, G. Verschaffelt, A. Georgievski, H. Thienpont, J. Danckaert, and I. Veretennicoff, Appl. Phys. Lett. 77, 1590 (2000).

[6] G. Verschaffelt et al., Proc. SPIE 3946, 246 (2000).

[7] T. Ackemann and M. Sondermann, Appl. Phys. Lett. 78, 3574 (2001).

[8] M.S. Miguel, Q. Feng, and J.V. Moloney, Phys. Rev. A 52, 1728 (1995).

[9] J. Martín-Regalado, F. Prati, M.S. Miguel, and N.B. Abraham, IEEE J. Quantum Electron. 33, 765 (1997).

[10] M.P.v. Exter, R.F.M. Hendricks, and J.P. Woerdman, Phys. Rev. A 57, 2080 (1998).

[11] S. Balle, E. Tolkacheva, M.S. Miguel, J.R. Tredicce, J. MartínRegalado, and A. Gahl, Opt. Lett. 24, 1121 (1999).

[12] D. Burak, J.V. Moloney, and R. Binder, Phys. Rev. A 61, 053809 (2000).

[13] M. San Miguel, S. Balle, J. Mulet, C. Mirasso, E. Tolkachova, and J.R. Tredicce, Proc. SPIE 3944, 242 (2000).

[14] J. Danckaert, B. Nagler, J. Albert, K. Panajotov, I. Veretennicoff, and T. Erneux, Opt. Commun. 201, 129 (2002).

[15] A.K.v. Doorn, M.P.v. Exter, and J.P. Woerdman, Appl. Phys. Lett. 69, 1041 (1996).

[16] R.F.M. Hendriks, M.P.v. Exter, J.P. Woerdman, A.v. Geelen, L. Weegels, K.H. Gulden, and M. Moser, Appl. Phys. Lett. 71, 2599 (1997).
[17] B. Ryvkin, K. Panajotov, A. Georgievski, J. Danckaert, M. Peeters, G. Verschaffelt, H. Thienpont, and I. Veretennicoff, J. Opt. Soc. Am. B 16, 2106 (1999).

[18] S. Balle, Phys. Rev. A 57, 1304 (1998).

[19] K.D. Choquette and R.E. Leibenguth, IEEE Photonics Technol. Lett. 6, 40 (1994).

[20] M.B. Willemsen, A.S.v.d. Nes, M.P.v. Exter, J.P. Woerdman, M. Kicherer, R. King, R. Jäger, and K.J. Ebeling, J. Appl. Phys. 89, 4183 (2001).

[21] H.F. Hofmann and O. Hess, Quantum Semiclassic. Opt. 10, 87 (1998).

[22] M. Sondermann and T. Ackemann (unpublished).

[23] M. Sondermann, report to COST action 286 on the results of a short term mission, 2002 (unpublished).

[24] L.A. Coldren and S.W. Corzine, Diode Lasers and Photonic Integrated Circuits (Wiley, New York, 1995).

[25] M.B. Willemsen, M.P.v. Exter, and J.P. Woerdman, Phys. Rev. A 60, 4105 (1999).

[26] J.-L. Vey, C. Degen, K. Auen, and W. Elsäßer, Phys. Rev. A 60, 3284 (1999).

[27] G. Giacomelli, F. Marin, and I. Rabbiosi, Phys. Rev. Lett. 82, 675 (1999).

[28] J. Mulet and S. Balle, IEEE J. Quantum Electron. 38, 291 (2002).

[29] J. Mulet and S. Balle, Phys. Rev. A 66, 053802 (2002).

[30] J. Mulet, C.R. Mirasso, S. Balle, and M. San Miguel, Proc. SPIE 4283, 139 (2001)

[31] P. Besnard, M.L. Charès, G.M. Stéphan, and F. Robert, J. Opt. Soc. Am. B 16, 1059 (1999).

[32] C. Masoller and N.B. Abraham, Phys. Rev. A 59, 3021 (1999).

[33] M. Sondermann, M. Weinkath, and T. Ackemann (unpublished).

[34] A.R. Cameron, P. Riblet, and A. Miller, Phys. Rev. Lett. 76, 4793 (1996). 
[35] Y. Ohno, R. Terauchi, T. Adachi, F. Matsukura, and H. Ohno, Phys. Rev. Lett. 83, 4196 (1999).

[36] A.K.v. Doorn, M.P.v. Exter, M. Travagnin, and J.P. Woerdman, Opt. Commun. 133, 252 (1997).

[37] E.L. Blansett, M.G. Raymer, G. Khitrova, H.M. Gibbs, D.K. Serkland, A.A. Allerman, and K.M. Geib, Opt. Express 9, 312 (2001).
[38] M. Sondermann, T. Ackemann, S. Balle, and J. Mulet (unpublished).

[39] T. Ackemann and M. Sondermann, Proc. SPIE 4286, 44 (2001).

[40] G. van der Sande, J. Danckaert, I. Veretennicoff, and T. Erneux, Phys. Rev. A 67, 013809 (2003). 